\title{
Dental Arch Monitoring by Splines Using 3d Digital Models
}

\author{
Nemanja V Majstorović ${ }^{1 *}$, Srdjan P Živković ${ }^{2}$ and Branislav R Glišić ${ }^{1}$ \\ ${ }^{1}$ Department of Orthodontics, Faculty of Dentistry, University of Belgrade, Military Technical Institute, Belgrade, Serbia \\ ${ }^{2}$ Coordinate Metrology Lab, Belgrade, Serbia \\ *Corresponding Author: Nemanja V Majstorović, Department of Orthodontics, Faculty of Dentistry, University of Belgrade, Military \\ Technical Institute, Belgrade, Serbia.
}

Received: November 06, 2019; Published: November 21, 2019

DOI: $10.31080 /$ ASDS.2019.03.0705

\begin{abstract}
Backrgound/Aim: Researchers in the field of dentistry have been conducting research into modelling and defining dental arches equitations. Nowadays, when 3D digital modelling is commonly utilized in dentistry, the approach to modelling, analysis and synthesis has changed. Clinical researches are related to aesthetic and functional analysis. The aim of this study was to increase repeatability and accuracy of defining and determining the coordinate system of the jaw as well as to defining mathematical criteria for monitoring and evaluating orthodontic treatment.

Methods: In this study, we used the plaster models of the jaw, optical scanner with structured light, 3D digital models, computer aided design (CAD) engineering tools adjusting the coordinate system, spline fitting of 3rd, 4th, 5th, 6th, 7th and 8th degrees.

Results: Splines of 3rd, 4th, 5th, 6th, 7th and 8th degrees were fitted from the initial state (K0) in all 10 successive controls (K1, K2, $\mathrm{K} 3, \ldots, \mathrm{K} 10)$. All splines were fitted through 12 points, from the right to the left side of the jaw: 6-5-4-3-2-1-1-2-3-4-5-6. Tabular and graphic presentations of the maximum and average deviation of dental arch fitting curves in successive controls were given.

Conclusion: The parameters of the maximum and average errors of fitting curves converge the dental arch values that are lower than the accuracy of the used optical scanners. The average error of fitting provides a general picture of the entire dental arch at each stage of treatment. Maximum error fitting points at a specified tooth where the largest deviation.
\end{abstract}

Keywords: Orthodontic; Computer-Aided Design; Dental Arch

\section{Introduction}

The application of new computer-aided technologies is nowadays found in various fields of dentistry: CAD - design and 3D modeling (prosthetics, orthodontics, endodontics), CAE - calculations and simulations of stresses and loads in the field of dentistry (teeth, jaws, joints) [2], respectively. using Finite Element Methods (FEM), then CAM - production of dental surcharges and orthodontic locks using computers, RP - rapid prototype production (fixed surcharges and elements of orthodontic appliances), CAQ - modeling and quality assurance of dental surcharges and orthodontic appliances, digital dentistry, digital dentistry all of the above approaches, and 'cloud' dentistry (a new concept for the application of ICT technologies in dentistry).

This paper presents the results of the study of tooth leveling in the occlusal plane, using the shape of the dental arch, where the monitoring of tooth leveling in the occlusal plane was performed using the shape of the dental arch, where a novelty was introduced in defining the function of the dental arch. Specifically, by applying a spline (a curved line defined by a step function), the initial and desired shape of the dental arch can be defined for the patient based on the master condition. The degree of this function, from third to eighth, is then determined, which best fits the investigated 
/ defined state. This means that the optimum function, or degree of splicing, is found using a least-squares technique that will fit or want to be achieved. Of course, the marking of points through which the deflection curve is defined is also made here using reference geometric entities (RGEs).

The state of research in the world

Research on the shape of dental arch has a long history of research in orthodontics, before the advent of 3D digital models. With the help of them, researchers have been provided with a powerful tool for simulating and analyzing complex mathematical models of multistage equations, which "describe" a dental arch.

One overview of the CAD / CAM system for generating and analyzing 3D digital models is presented in [1], and it provides the first systematic overview of hardware / software for these purposes. Advantages of these approaches in comparison to classical systems are given. One of the first analyzes using 3D digital models of dental arch shape, as a basic step in patient malocclusion analysis, with measurement and evaluation of various dentition parameters such as: occlusion, mesiodistal tooth width, Bolton ratio, arc length, arch width, incisal flap and the incisal spacing is given in [2]. Here are examples of several programs used for these analyzes.

The measurement and analysis of PAR indices on 3D digital models with a comparison of the accuracy of this parameter on gypsum models is presented in [3]. This reliable method for evaluating malocclusion has been investigated in 48 models. PAR index analysis was performed by determining reliability and validity, using analysis of variance and correlation coefficient for these two types of models. 3D digital models have been found to provide valid and reliable occlusion parameters, with greater accuracy.

A specific example of the study was presented in [4] for comparisons of the shape of the dental arch for the tooth axis with respect to its crown and root tip, class 1 and 2, group 1 malocclusion, using WALA points, on a sample of 35 class 1 patients and 32 Class 2 patients.

The results obtained show that the shape of the lower arch is the same for both classes. Also, there is no difference in tooth arch shape for both basal bone groups. Finally, using WALA points can successfully plan orthodontic therapy.
Paper [5] presents an analysis of the ideal shape of the dental arch, a 4 degree polynomial, on a sample of 15 patients. This model has been found to be good, but only when the coordinates of all the teeth in the lower and upper jaws are known. In the event that the patient lacks a tooth (especially from the first molar to the central incisor), then the $\beta$ function is a better solution for simulating the shape of the dental arch.

In [6], the results of a study, which relate to the new classification of dental arch shape at normal bite, obtained using 3D digital models, are presented. Imprints of 115 young patients, lower and upper jaw, were taken, wearing an orthodontic appliance. A cluster analysis of the classification of the shape of the dental arch with widths was made: between canines, premolars and molars. The first set generated a subset of 68 prints, which were divided into 4 clusters, based on the orthodontic parameters of the anterior and posterior teeth. This demonstrates that 3D analysis can be used in a new way.

A comparison of the accuracy of gypsum, 3D models, and reconstructed 3D models is presented in [7]. The null hypothesis was - there is no difference in the accuracy of the models defined in the above three ways. The sample included 30 randomly selected gypsum models, on which a total of 16 orthodontic parameters were measured in three planes, twice with a span of seven days

The reproducibility of the measurements was investigated by Bland-Altman analysis, and the reliability of the results obtained was tested with the non-parametric Wilcoxon Signed Rank, as the analysis of the measurement data showed that the obtained measurement results do not follow the normal law of distribution. The largest difference in measurement results was obtained with manual measurement, ( $p>0.2$ ), while it decreases as we go from reconstructed to $3 \mathrm{D}$ digital models, where the repeatability of the results is almost absolute and tends to zero.

The main measurement difference in all planes was $0.14 \mathrm{~mm}$ $(\mathrm{SD}=0.1 \mathrm{~mm})$ and was not statistically significant $(\mathrm{p}>0.2)$. Measurements made in the $x$ and $y$ planes were not statistically significant for the reconstructed and gypsum models $(\mathrm{p}>0.3)$ or $3 \mathrm{D}$ digital models $(p>0.5)$. However, in the z-plane, the measurement differences were significant, $\mathrm{p}<0.0001$. All measurements of 3D digital models in the z-plane were significantly smaller than those of the corresponding plaster and reconstructed models. 
The above analyzes showed all the benefits of 3D digital models. Investigation of the accuracy of measurements on classical prints (scales), 3D models and models obtained by CT scanning on 34 models is presented in [8]. Measured: mesiodistal width of tooth, width of arch, length of arch, other parameters of arch and depth of palate. The following results were obtained: mesiodistal distance at the upper jaw between the right and left premolars, left central incisor, and right first molar. The lower jaw measured the distance of the left and right incisors, the right canine and the left first molar. Satisfactory accuracy of all three methods was obtained, in the following order, from least to largest: conventional, CT, 3D model.

The study presented in [9] showed the forms of dental arch, obtained by factor analysis of 137 digital models, all three classes of bites (43, 50 and 44 prints), young people, between 15 and 18 years, 71 girls and 66 boys. The main elements of factor analysis were: the ratio of width / height of the lower canine, accounting for $82.8 \%$ of variability $(\mathrm{p}<0.001)$. The width of the dental arch in the front part for class III is smaller compared to class II. A similar finding applies to Class I with respect to Classes II and III. The homogeneity of the results describing dental arch shapes by classes is: $61.1 \%$ Class III, $57.8 \%$ Class II and 52.4\% Class I. Also, the influence of sex on dental arch shape has not been shown to be significant.

The conclusion is that the shape of the dental arch and its dimensions change from class to class. The shape of the dental arch in the upper jaw varied significantly in the posterior segment of the dental string, while with the lower jaw the largest changes were observed in the anterior segment of the dental string. In patients with class III, the shape of the dental arch is easy to determine, while the reverse is the case with patients of class I. The research presented in [10] presented a combined method of determining dental arch by superimposing in the lower jaw, using two techniques - CBCT and 3D modeling. The sample investigated for this problem was 30 prints, and two methods of superposition were applied. Using CBCT images, the entire structure of the tooth (including the root) was monitored, and the 3D model monitored the change of position of the 10 contact points in the bite plane, as well as the determined length of the dental arch. The final research results show that this method is simpler and more reliable for determining the length of the dental arch.

Comparison of the accuracy of three-dimensional models obtained by intra-oral scanner, 3D digital model and conventional measurement is presented in [11]. The study was performed on 35 samples / patients, and accuracy was measured by overlapping 3-dimensional models for both groups. Statistical significance was assessed by t-test (alpha $=0.05$ ). The obtained dental arch length for $3 \mathrm{D}$ models is: accuracy - $1.6 \pm 0.6 \mu \mathrm{m}$, veracity $-5.3 \pm 1.1 \mu \mathrm{m}$. The same parameters for the intra-oral scanner are: $12.5 \pm 2.5 \mu \mathrm{m}$ and $20.4 \pm 2.2 \mu \mathrm{m}$, and for conventional measurements are: 32.4 $\pm 9.6 \mu \mathrm{m}$ and $58.6 \pm 15.8 \mu \mathrm{m}$. For all these analyzes, $\mathrm{p}<0.001$, was the same. The conclusion is that $3 \mathrm{D}$ modeling produces the most accurate and true results.

The study [12] concerned measurements on 3D models and gypsum prints of a patient undergoing orthodontic treatment, 7 parameters of the dental arch (width / distance of the first 6 teeth, left and right in the upper jaw), on a sample of 27 patients. Statistical analysis was performed using ANOVA and t-test pair. The results show that there is no statistically significant difference between the models for the width and depth of the dental arch, reducing the difference between the anterior and posterior teeth from $2.3 \mathrm{~mm}$ to $2 \mathrm{~mm}$. However, significant statistical differences were obtained for the folding parameter, which indicates the effect of locks on 3D models. The conclusion is that the flap parameter should be taken into account and measured in the future, taking into account the position of each tooth individually.

Finally, the latest exploration of the shape of the dental arch is presented in [13]. It is a study that followed children aged 14 to 18 with class III malocclusion in the upper and lower jaws. The $t$ test showed a high correlation between the width of the molars in the lower / upper jaw and the shape of the dental arch for the investigated sample of 20 prints, using 3D digital models. At the end of this analysis, we can conclude that measuring, analyzing, and simulating the shape of a dental arch using a 3D model is a new and very powerful tool for the clinician's orthodontist to do his or her work and research.

\section{Dental arch circumference - our research}

In this study, 155 prints were scanned from the Clinic for Fork Orthopedics, School of Dental Medicine,Belgrade, on the Next Engine scanner. They were selected at random, and 3D modeling was performed using Siemens PLM NX10 software [14 -16].

For the interpolation (fitting) of curved lines that describe the dental arch, this software was used because of its flexibility as well as the openness of the architecture, which allows it to be easily applied 
in very different areas. Its advantage over specialized software packages in the field of orthodontics is in sophisticated modules specializing in free-form, sculptured surfaces, such as teeth and their anatomy. It is a fact that modern medicine and dentistry are unthinkable without the cooperation of mechanical engineers and dentists. This study is precisely an example of the interdisciplinary application of engineering modeling in orthodontics.

In the existing literature, there is no extensive mathematical analysis of the function of dental arch shape in persons with normal occlusion, with orthodontic therapy. Therefore, the aim of this part of the research is to define the function of the dental arch as a polynomial, from 3rd to 8th degree, and perform its detailed analysis, following all stages of orthodontic therapy. The essence of the problem is that during therapy, the position of the tooth in the jaw changes, which leads to a change in the position of the coordinate system of the jaw, in relation to which the function of the dental arch is modeled. As a consequence, the shape of the dental arch for the two conditions - the stages of therapy - can be compared with relatively low accuracy. For these reasons, the GOKS was determined here by an advanced ABO method, thereby canceling out this negative impact, so that the dental arch can be compared exactly for each phase of orthodontic therapy with each other.

The curved lines describing the dental arch are defined in the X-Y (occlusal) plane of the orthodontic coordinate system of the digital jaw model. It is determined according to $\mathrm{ABO}$ recommendations [18], which we have upgraded according to the procedure in point 5.4 , and this manual is the defacto generally accepted world standard for setting a coordinate system on a digital model. It is generated as a. ply,. stl, or. obj file, and contains all the elements for further analysis. According to this instruction [18], the resolution of the scanners on which 3D digital models are generated must be $0.10 \mathrm{~mm}$ or greater and an accuracy of $0.20 \mathrm{~mm}$ or greater. One of the basic features of the GOKS determination procedure is that it provides repeatability for measuring (scanning) one fork model on different scanners, which is extremely important for 3D digital models. On the other hand, when comparing several different jaw models, which is the case during one patient's orthodontic therapy, the $\mathrm{ABO}$ instruction does not provide sufficient accuracy. First of all, this refers to defining the orientation of the Y-axis (Y-Z: medial plane) as well as the origin (origin) of the coordinate system
Figure 1 shows a 3D model with anatomical points on the teeth, from 1 to 6, left and right, as well as shapes of splines from the third to the eighth degree.

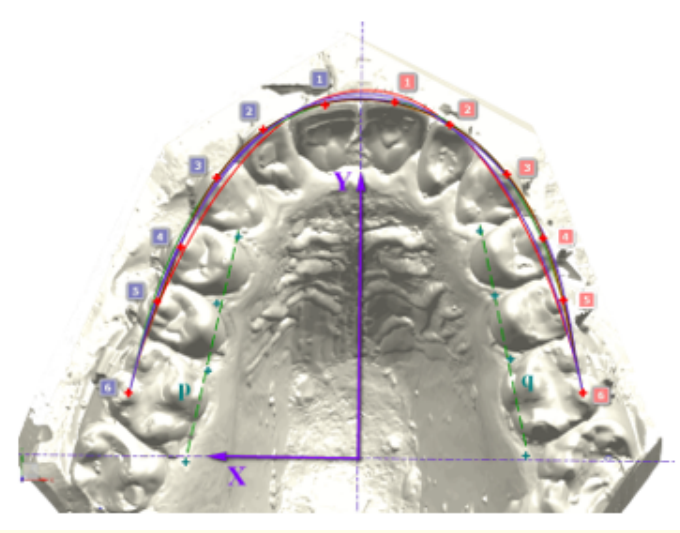

Figure 1: Tooth Onion - Grade 3 to 8 spines.

The analysis of the use of splines of $3,4,5,6,7$, and 8 degrees in modeling the curve of the dental arch line. The mathematical foundations [18] of this model are splines (curved lines), which are widely used in various, not just engineering fields. Using them on CAD / CAM / CAE is simple and intuitive, and there is no modern CAD / CAM / CAE system that doesn't have the ability to create splines.

When modeling the dental arch curve, in these studies, the first dilemma that arises is - what degree of curve line / spline to choose? The earliest description of the ideal dental arch was provided by Bonwill (1887) [19] who suggested that the ideal dental arch was based on an equilateral triangle with a base representing width in the condyle region. A little later Hawley (1905) supplemented this model, which, as a Bonwill-Hawley dental arch, dominated orthodontics, and was most applicable by manufacturers. Black (1902) described that the teeth in the upper jaw were located in a semi-ellipse shape, while Angle (1907), on the other hand, considered his " occlusion line " to be represented by a parabolic curve [19].

The application of computers has brought a new approach to solving this problem. BeGole and Lyew (1998) and Davis and BeGole (1998) used a third-degree function to model the shape 
of dental arches. The use of the cubic equation has been shown to be helpful in evaluating dental arch changes during and after orthodontic therapy [19]. These surveys are focused on personal orthodontics, one that relates to a specific person rather than analyzing the crooked dental arch line as the average of a section or a wider population. The case study presented here showed that Grade 6 spline most naturally described the curve of the dental arch at all stages of therapy, figure 2. After the eighth degree, there is no point in modeling the dental arch, because the 8 and 9 degree splines coincide.

The splines of 3, 4, 5, 6, 7 and 8 degrees from the initial state (master model) were interpolated in all 10 successive controls. All splines are fit through 12 points, from right to left side of fork: 6-5-4-3-2-1-1-2-3-4-5-6. The interpolation points are indicated by red crosses in Figure 6.4.2. Each of the interpolated splines at the beginning has a slope (End Slope) of straight lines "p" and at its end has a slope (End Slope) of straight lines "q" shown in Figure 6.3.2. The symmetry of the lines "p" and "q" determines the direction of the $\mathrm{Y}$ axis as explained in the chapter "Coordinate system of the digital fork model".
For each of the fitting splines, the CAD / CAM / CAE system provides the following information: Maximum Fitting Error (M), maximum point error indicator, and Average Fitting Error (A). In Figure 6.4.2, the point with maximum deviation is marked with a red circle. The results obtained are shown in table 1

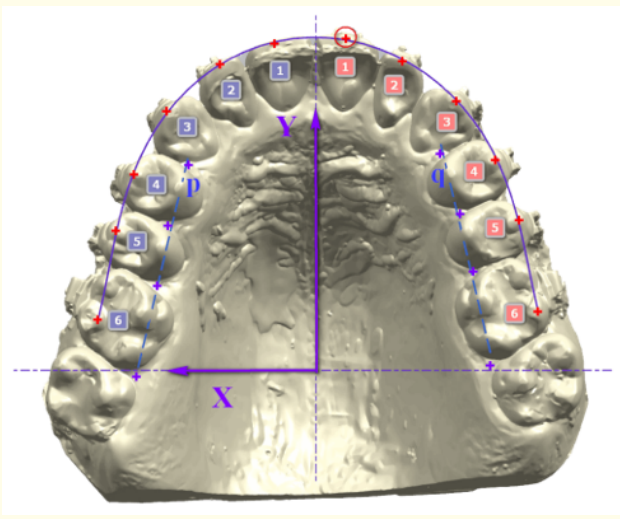

Figure 2: Tooth arch - 6th degree spline (10th control) for the upper jaw.

\begin{tabular}{|c|c|c|c|c|c|c|c|c|c|c|c|c|}
\hline $\begin{array}{l}\text { Spline } \\
\text { degree }\end{array}$ & & KO & K1 & K2 & K3 & K4 & K5 & K6 & K7 & K8 & K9 & K10 \\
\hline \multirow{2}{*}{3} & $\mathbf{M}$ & 2,6419 & 2,0781 & 1,7265 & 0,8201 & 0,9267 & 1,6789 & 1,1882 & 1,8624 & 2,5292 & 2,1747 & 1,8950 \\
\hline & $\mathbf{A}$ & 1,2435 & 0,8817 & 0,9343 & 0,2849 & 0,3718 & 0,5465 & 0,4455 & 0,9098 & 1,0761 & 1,1991 & 0,8907 \\
\hline \multirow{2}{*}{4} & $\mathbf{M}$ & 2,0158 & 1,8785 & 1,8311 & 0,6814 & 0,9350 & 1,2321 & 1,5078 & 2,2048 & 2,4748 & 2,4857 & 2,1859 \\
\hline & A & 0,9762 & 0,8283 & 0,7214 & 0,3224 & 0,4361 & 0,6122 & 0,6731 & 1,0754 & 1,2357 & 1,3144 & 1,0987 \\
\hline \multirow{2}{*}{5} & $\mathbf{M}$ & 1,7169 & 1,6267 & 1,3368 & 0,8298 & 0,7072 & 0,3968 & 0,6918 & 0,6134 & 0,8038 & 0,4946 & 0,2706 \\
\hline & A & 0,8106 & 0,7257 & 0,7366 & 0,2527 & 0,2086 & 0,1994 & 0,2425 & 0,1848 & 0,2271 & 0,2415 & 0,1064 \\
\hline \multirow{2}{*}{6} & $\mathbf{M}$ & 1,6145 & 1,4461 & 0,8584 & 0,5662 & 0,3757 & 0,4436 & 0,4041 & 0,3117 & 0,5306 & 0,4354 & 0,2471 \\
\hline & $\mathbf{A}$ & 0,7019 & 0,6228 & 0,3463 & 0,2312 & 0,1316 & 0,2070 & 0,1993 & 0,1037 & 0,1684 & 0,2017 & 0,0968 \\
\hline \multirow{2}{*}{7} & M & 1,5095 & 1,3076 & 0,6270 & 0,3636 & 0,1725 & 0,2617 & 0,2516 & 0,2264 & 0,5382 & 0,4064 & 0,2191 \\
\hline & A & 0,6513 & 0,6219 & 0,2597 & 0,0994 & 0,0742 & 0,1122 & 0,0957 & 0,0860 & 0,1722 & 0,1947 & 0,0911 \\
\hline \multirow{2}{*}{8} & M & 1,3729 & 1,2245 & 0,4834 & 0,3595 & 0,0790 & 0,2531 & 0,2081 & 0,2548 & 0,5441 & 0,4360 & 0,2060 \\
\hline & A & 0,7106 & 0,6296 & 0,2127 & 0,1031 & 0,0420 & 0,1149 & 0,0910 & 0,0767 & 0,1801 & 0,1798 & 0,0994 \\
\hline
\end{tabular}

Table 1: Upper Fork - Maximum (M) and Average (A) Deviation of Fit Splines from Grade 3 to Grade 8.

Legend: $\mathrm{K}$ - control (0-start condition, 10th control). M - max parameter values in the observed control. A - average value of the same parameter. All values are in $\mathrm{mm}$. 
Diagrammatic analysis of max deviation is shown in figure 3 . The values on the $\mathrm{y}$-axis are in $\mathrm{mm}$ and analyzes are given for splines of 3 to 8 degrees. The figure above shows that the splines of the seventh and eighth degrees almost coincide.

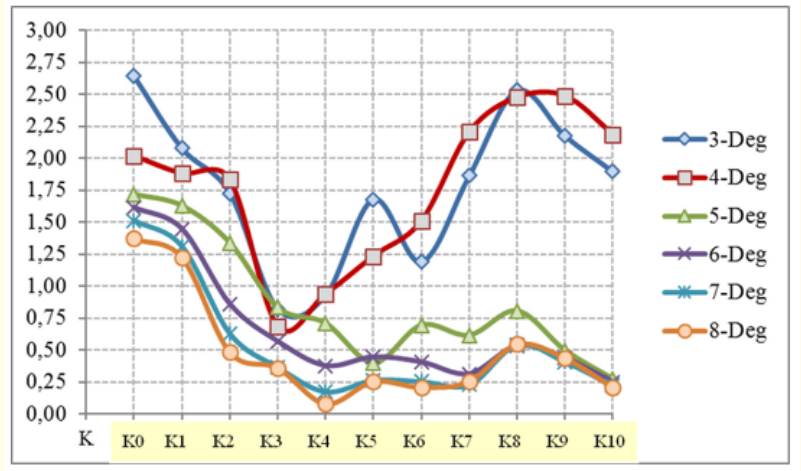

Figure 3: Diagram of maximum deviations.

Legend: The y-axis values in mm for max deviations of splines of varying degrees in controls from the master model (K0) to the last - K10 control. Axis x - control, from master (K0) to tenth (K10).

Diagrammatic analysis of average deviations is shown in figure 4. The values on the $y$-axis are in $\mathrm{mm}$ and analyzes are given for splines of 3 to 8 degrees. The figure above shows that the splines of the seventh and eighth degrees almost coincide.

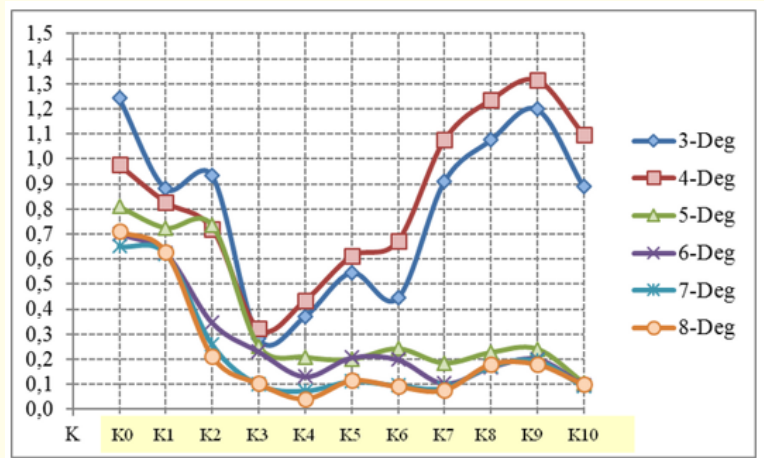

Figure 4: Diagram of average deviations.

Legend: The y-axis values in $\mathrm{mm}$ for average deviations of splines of varying degrees in controls from the master model (K0) to the last - K10 control. X axis - control, from master (K0) to tenth (K10).

\section{Discussion}

The researches analyzed in the previous point, led to the conclusion that the shape and characteristics of the dental arch, except in [20], were considered in the context of the functional characteristics of the jaw and the position of the tooth in it, which is also the primary task of clinical orthodontics. However, thanks primarily to ICT technologies, today we can go a step further, to make these analyzes more subtle, which means that a far greater number of parameters will be obtained that will describe and define it. Thus, in [21] it was suggested that in order to ideally describe the dental arch curve, a higher degree polynomial must be used. The curve (function) of the dental arch should have considerable flexibility, meaning that it can be adjusted to fit any size of dental arch, and may include asymmetries of the jaw, if any. It is also important to emphasize the fact that the dental arch is an imaginary curve, which means that it is descriptive information, that is, a function, representing a set of discrete points. Therefore, the mathematically obtained curve of the dental arch should be adjusted according to individual points, on each tooth $[22,23]$.

This approach represents a curve adjustment or its interpolation. Curve adjustment is a concept that serves to adjust the mathematical shape of the curve according to the curve of the actual shape of the dental arch. The curve generated by the proposed mathematical functions is adjusted according to the markings on the teeth that are believed (the doctor determines) to reliably define the dental arch. In order to determine the best mathematical function that would describe the curves of the dental arch, several authors tested different mathematical functions (models) that would best fit this purpose. Some of these models are: conical curve shapes, $U$ curves, cubic equations, second to eighth degree equations, mixed models and beta function $[24,25]$. Lastly, it is difficult to generalize their findings or draw conclusions for many reasons: different goals, different study samples with different criteria, and different methodology.

Accurate determination of the global jaw coordinate system as well as its precise repeatability $[17,26]$ is key to defining the curve line describing the dental arch. The error that is made in the initial step of determining the coordinate system becomes systematic and cannot be eliminated. If the fork coordinate system is not precisely defined in all successive controls then these controls cannot be compared reliably. The conclusions drawn about the progress of orthodontic therapy may be completely wrong. 
The importance of correctly defining the coordinate system is very large. Its position and direction of the plane must be reproducible. It further depends on how the tooth movements between the two controls can be compared. Even the smallest setup error can lead to incorrect results. Not only will the results be unclear, but there may be misconceptions.

Another very important thing is defining and setting up the RGE $[17,26]$. They must be placed in the same place on each 3D model. Setting by free belief and "by eye" does not produce accurate results. The mistake of setting up only one entity entails hard-tocorrect mistakes.

The maximum and average deviations for splines 6,7 , and 8 degrees converge values less than $0.1 \mathrm{~mm}$, which is below the minimum required accuracy prescribed by ABO. Grades 3 and 4 are not appropriate for describing this patient's dental arch curve.

The Grade 6 spline looks the most natural in all stages of treatment, and corresponds to the best leveling of the teeth, for the 10th control of Figures 6.4.2.

It is important to note here that the developed model allows the following: that the doctor orthodontist predicts the degree of splicing (dental arch circumference), at the beginning of therapy, and according to the treatment plan of the patient, or in each control to check his degree (shape), depending on the level achieved. leveling teeth.

The latter approach was used in these studies.

At all stages of the treatment, the dentist has the correct information on which tooth to pay the most attention because his / her point (tooth) with maximum deviation from the fit curve is indicated.

It may be more appropriate for another patient to have different splines of different degrees than this patient because it is individual (gender, age, race...), which is another confirmation of the hypothesis of personal orthodontics from point 3 of this thesis.

On the other hand, the prediction of the shape of the dental arch can be made, in accordance with the plan of the therapist's orthodontist, which this model can accomplish.

\section{Conclusions and future research}

The analysis of tooth leveling through research on the shape of the arch, presented in this paper represents the leveling of teeth in the $x-y$ plane (occlusal plane), is a new approach, the application of splines for the analysis and description of the shape of the dental arch. And this is provided by the Reference Geometric Entities (RGE) [17,20], from the curve line class, which is done here. Sixthdegree spline has been shown to best describe the dental arch from a physician's perspective.

Therefore, the paper describes the developed and applied model of reference geometric entities of RGE through the planning and monitoring of orthodontic therapy, for tooth leveling, but with determined values of position change (the paper shows translation in three planes or along three axes, and for the next research will be tooth rotation included).

\section{Bibliography}

1. Waldemar P. "Digital impressions and handlig of digital models: the future of dentistry". Dental Press Journal of Orthodontics 15.5 (2010):18-22.

2. Quimby M., et al. "The Accuracy and Reliability of Measurements Made on Computer-Based Digital Models". Angle Orthodontics 74 (2004): 298-303.

3. Mayers M., et al. "Comparison of peer assessment rating (PAR) index scores of plaster and computer-based digital models". American Journal of Orthodontics and Dentofacial Orthopedics 128.4 (2005): 431-434.

4. Ball R., et al. "Comparison of dental and apical base arch forms in Class II Division 1 and Class I malocclusions". American Journal of Orthodontics and Dentofacial Orthopedics 138 (2010): 41-50.

5. Adaškevičius R and VasiliauskasA. "Evaluation of Dental Arch Form Using 3D Dental Cast Scanning Technology, Journal of Electronics and Electrical Engineering". Medicine Technology 5.93 (2009): 99-103.

6. Park K., et al. "New classification of lingual arch form in normal occlusion using three dimensional virtual models". The Korean Journal of Orthodontics 45.2 (2015):74-81.

7. Keating A., et al. "Acomparison of plaster, digital and reconstructed study model accuracy". Journal of Orthodontics 35 (2008): 191-201. 
8. Mohammed El-Zanaty H., et al. "Three-dimensional dental measurements: An alternative to plaster models". American Journal of Orthodontics and Dentofacial Orthopedics 137 (2010): 259-265.

9. Slaj M., et al. "Discriminant factor analysis of dental arch dimensions with 3-dimensional virtual models". American Journal of Orthodontics and Dentofacial Orthopedics 140 (2011): 680-687.

10. KeePark T., et al. "A method for mandibular dental arch superimposition using 3D cone beam CT and orthodontic 3D digital model". The Korean Journal of Orthodontics 42.4 (2012):169181.

11. Eder A and Mehl A. "Accuracy of complete-arch dental impressions: A new method of measuring trueness and precision". Journal of Prosthetic Dentistry 109 (2013):121-128.

12. Kooka Y., et al. "Overjet at the Anterior and Posterior Segments: Three-Dimensional Analysis of Arch Coordination". Angle Orthodontist 79 (2009): 495-501.

13. Majstorović, N., et al. "Dental Arch Monitoring by Splines Fitting Error During Orthodontic Treatment Using 3D Digital Models". Časopis Vojnosanitski Pregled 76.3 (2019): 142-148.

14. NN ATOS Manuel for user (2016).

15. NN. Manuel for user (2016).

16. Majstorović N., et al. Referentni geometrijski entiteti, Stomatološki glasnik Srbije 61.2 (2014):102-112.

17. The American Board of Orthodontics (ABO) Digital Model Requirements, Original Release 04 (2013): 23.

18. Živković S. "Coordinate metrology in manufacturing of the complex spatial forms with applications to the aerodynamic surfaces, Monographic series: Scientific-technical information, Vol. LI No.2, Published by Military Technical Institute, ISBN 978-86-81123-68-3 (2014).

19. Adaškevičius R and Vasiliauskas A. "Evaluation of Dental Arch Form Using 3D Dental Cast Scanning Technology" Journal of Electronics and Electrical Engineering, Medicine Technology5.93 (2019): 99-103.

20. Harbia S., et al. "Mathematical analyses of dental drch curvature in normal occlusion". Angle Orthodontist 78.2 (2008): 281-287.
21. Noroozi H., et al. "The dental arch form revisited". Angle Orthodontist 71.5 (2001): 386-389.

22. Muhamad A., et al. "The curve of dental arch in normal occlusion”. Open Science Journal of Clinical Medicine 3.2 (2015): 4754.

23. Pokhariyal G. "Humans dental arch shapes, Global Journal of Medical Research". Jouranl of Dentistry and Otolaryngology 15.4 (2015): 1-4.

24. Zhang Y., et al. "Motion control point optimization of dental arch generator". International Journal of $u$ - and e-Service Science and Technology 6.5 (2013): 49-56.

25. Majstorović N., et al. "Dental Arch Monitoring by Splines Fitting Error During Orthodontic Treatment Using 3D Digital Models". Časopis Vojnosanitski Pregled 76.3 (2019):142-148.

\section{Volume 3 Issue 12 December 2019}

(C) All rights are reserved by Nemanja V Majstorović., et

al. 